\title{
Correction to: Fundamentals of High Frequency CMOS Analog Integrated Circuits
}

\section{Correction to:}

D. Leblebici, Y. Leblebici, Fundamentals of High Frequency CMOS Analog Integrated Circuits, https://doi.org/10.1007/978-3-030-63658-6

The original version of this 2nd edition book was unfortunately published with errors in Figure 1.34, Figure 1.41, Figure 3.39, Figure 3.40, Figure 3.41, Figure 3.42, Figure 3.54, Figure 4.2, Figure 4.30, Figure 4.39, Figure 6.1, Figure 6.14 and Expression 5.20. This has now been corrected and an erratum to this book has been published.

\footnotetext{
The updated online versions of these chapters can be found at https://doi.org/10.1007/978-3-030-63658-6_1 https://doi.org/10.1007/978-3-030-63658-6_3 https://doi.org/10.1007/978-3-030-63658-6_4 https://doi.org/10.1007/978-3-030-63658-6_5 https://doi.org/10.1007/978-3-030-63658-6_6

(C) The Author(s), under exclusive license to Springer Nature Switzerland AG 2021 
The corrected figures and expressions are given below.

\section{Chapter 1}

Fig. 1.34 (page 41)

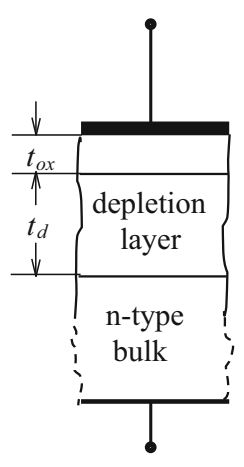

(a)

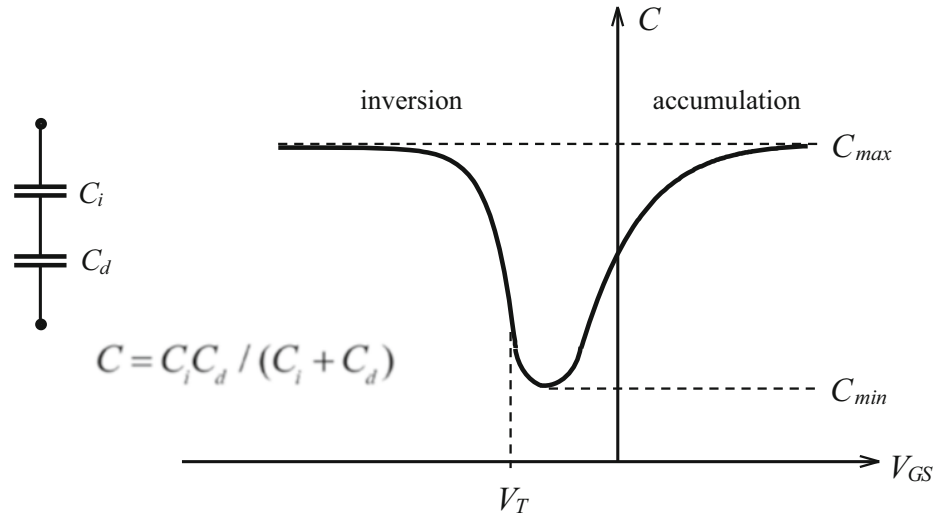

(b)

Fig. 1.41 (page 47)

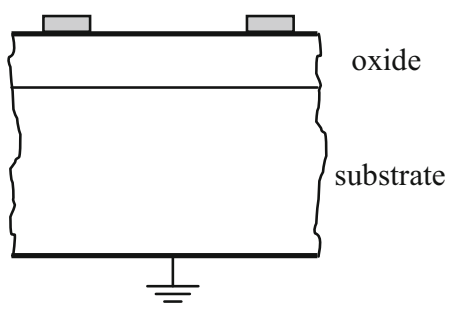

(a)

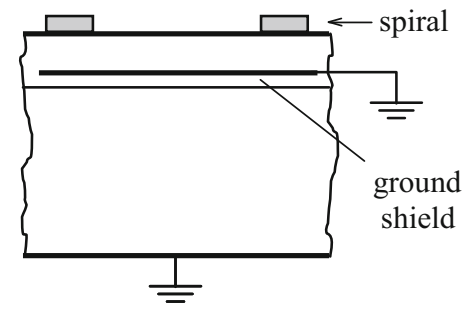

(b)

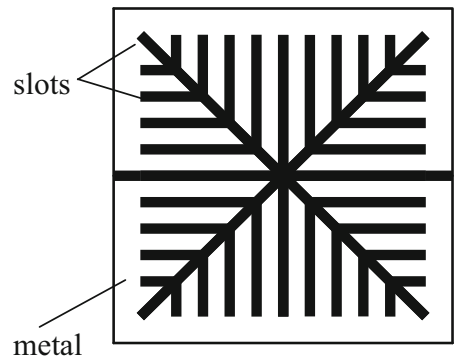

(c) 
8 Correction to: Fundamentals of High Frequency CMOS Analog Integrated Circuits

CB

Chapter 3

Fig. 3.39 (page 146)

(a)

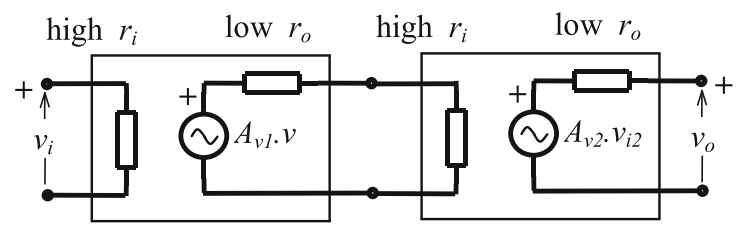

$$
A_{v T} \simeq A_{v 1} \times A_{v 2} \quad \text { for } \quad r_{i 2} \gg r_{o 1}
$$

(b)

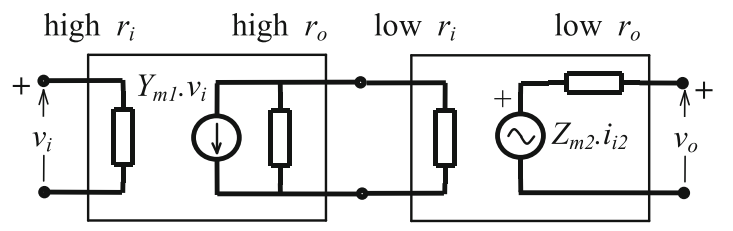

$$
A_{v T} \simeq-Y_{m 1} \times Z_{m 2} \quad \text { for } \quad r_{i 2} \ll r_{o 1}
$$

Fig. 3.40 (page 147)

(a)

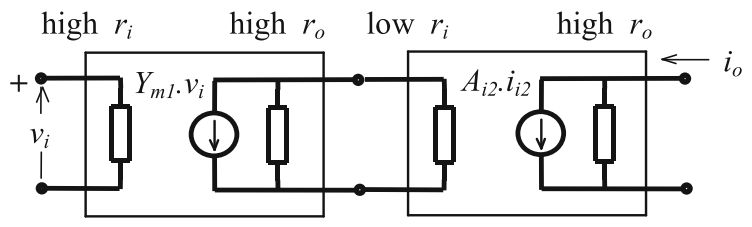

$$
Y_{m T} \simeq-Y_{m 1} \times A_{i 2} \quad \text { for } \quad r_{i 2} \ll r_{o 1}
$$

(b)

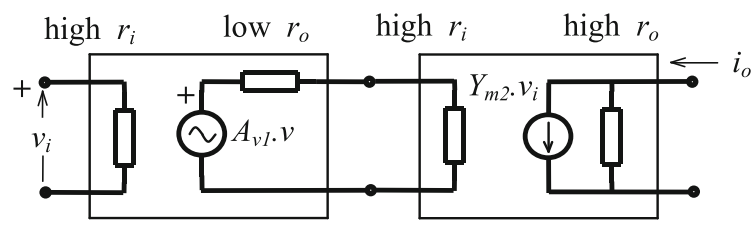

$$
Y_{m T} \simeq A_{v 1} \times Y_{m 2} \quad \text { for } \quad r_{i 2} \gg r_{o 1}
$$


Cu

8 Correction to: Fundamentals of High Frequency CMOS Analog Integrated Circuits

Fig. 3.41 (page 147)

(a)

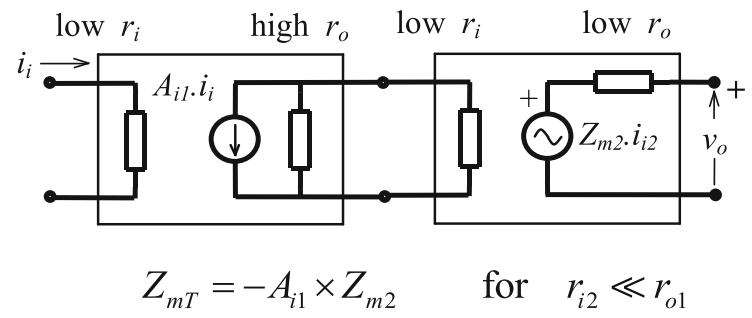

(b)

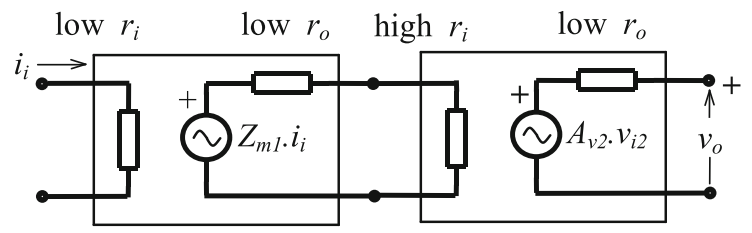

$$
Z_{m T}=Z_{m 1} \times A_{v 2} \quad \text { for } \quad r_{i 2} \gg r_{o 1}
$$

Fig. 3.42 (page 148)

(a)

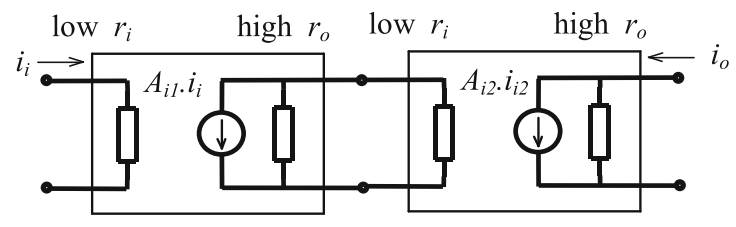

$$
A_{i T}=-A_{i 1} \times A_{i 2} \quad \text { for } \quad r_{i 2} \ll r_{o 1}
$$

(b)

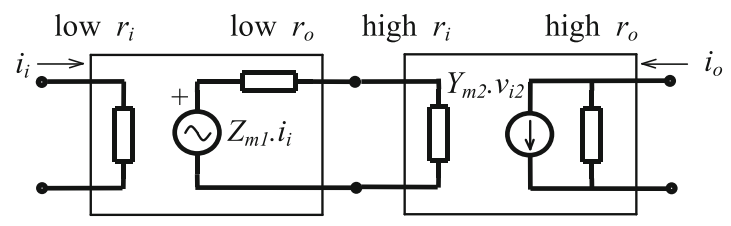

$$
A_{i T}=Z_{m 1} \times Y_{m 2} \quad \text { for } \quad r_{i 2} \gg r_{o 1}
$$


Fig. 3.54 (page 161)

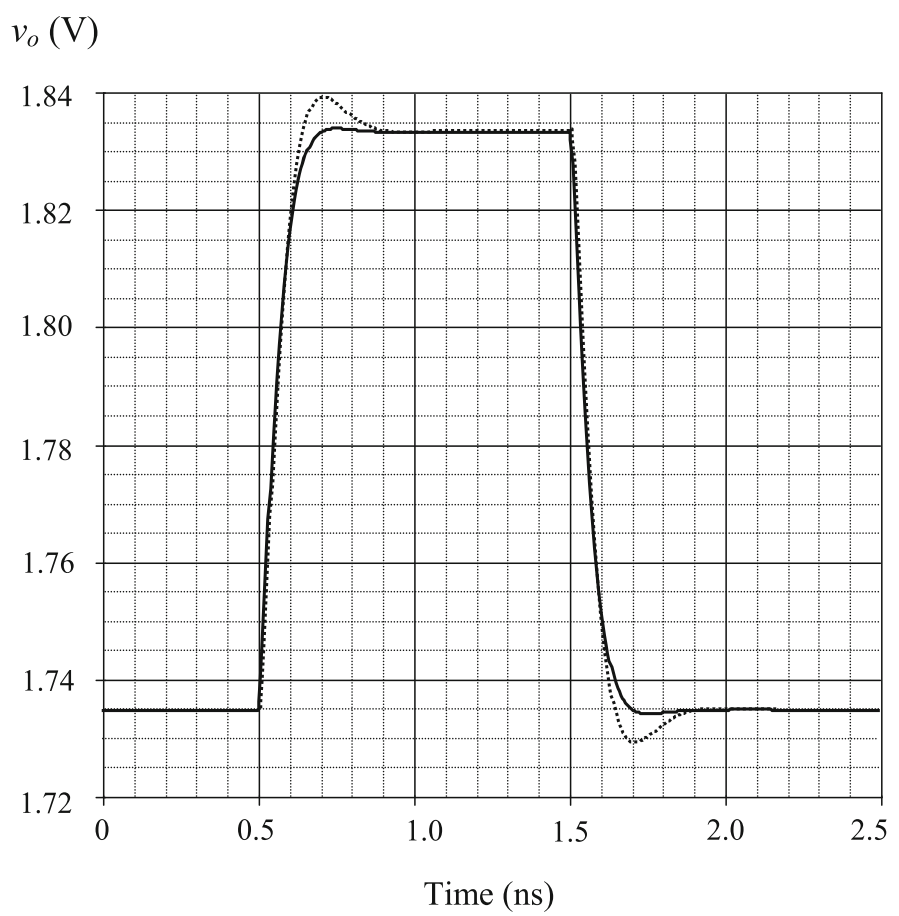

\section{Chapter 4}

Fig. 4.2 (page 168)
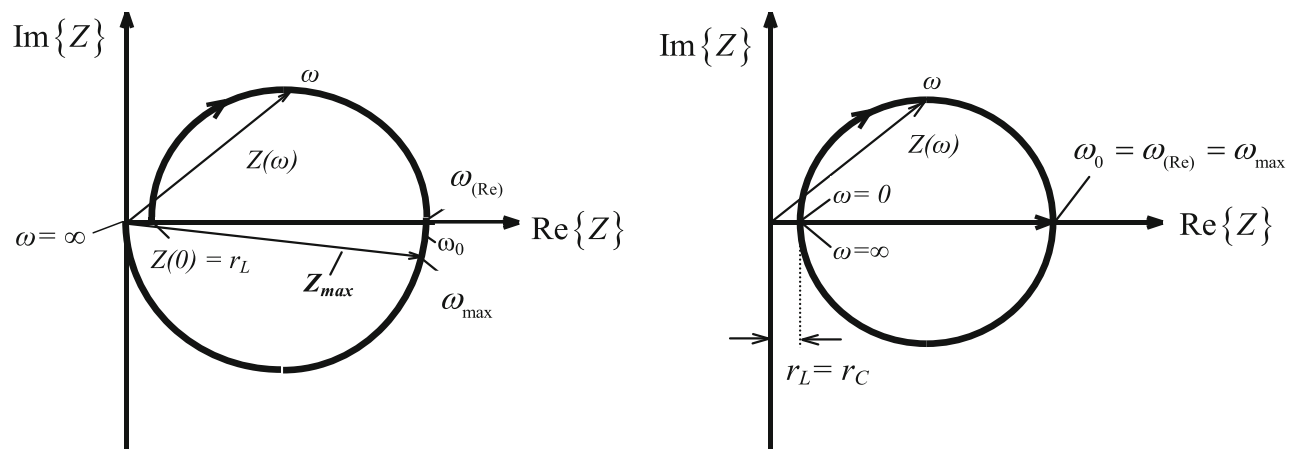
Fig. 4.30 (page 206)

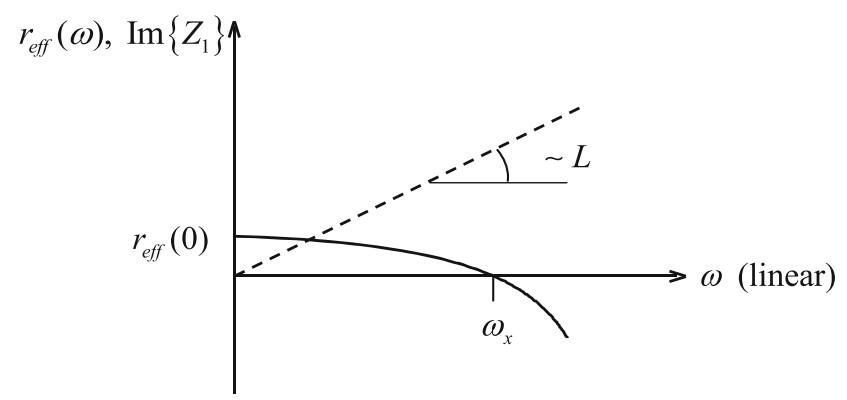

Fig. 4.39 (page 215)
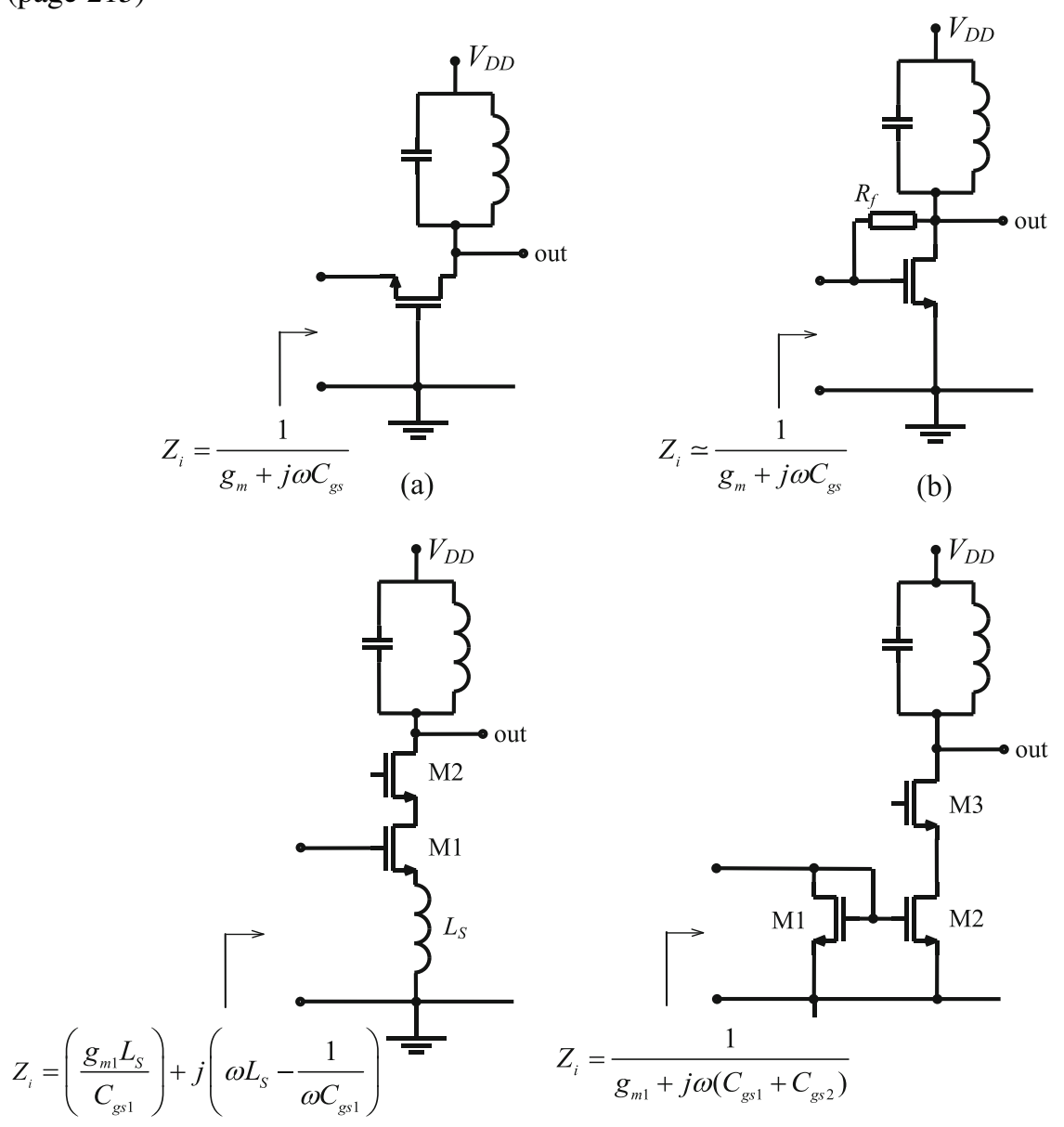

(c)

(d) 


\section{Chapter 6}

Fig. 6.1 (page 270)

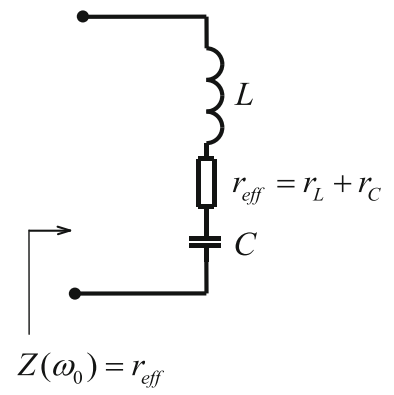

Fig. 6.14 (page 284)

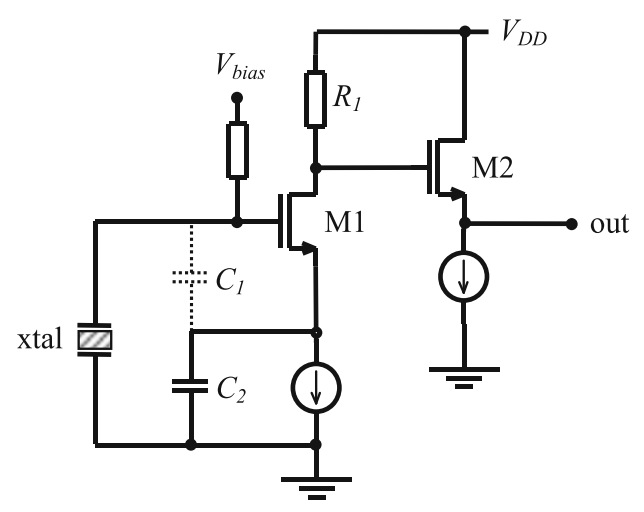

\section{Chapter 5}

Expression (5.20) (page 224)

$$
\bar{i}_{n d T}=\bar{i}_{n d} \frac{1}{\left(1+g_{m} R_{S}\right)+\frac{R_{D}+R_{S}}{r_{d s}}} \cong \bar{i}_{n d} \frac{1}{\left(1+g_{m} R_{S}\right)}
$$

